\title{
Coupling ALE Technique and Harmonic Parametrization to Describe Concurrent and Successive Elementary Cell Deformations
}

\author{
Rachele Allena (Corresponding author) \\ Ecole Centrale Paris \\ Grande Voie des Vignes, 92295 Châtenay-Malabry, France \\ Tel: 33-141-131-321 E-mail: racheleallena@gmail.com \\ Denis Aubry \\ Ecole Centrale Paris \\ Grande Voie des Vignes, 92295 Châtenay-Malabry, France \\ Tel: 33-141-131-321Ｅ-mail: denis.aubry@ecp.fr
}

Received: September 28, 2011

Accepted: October 24, 2011 Published: December 31, 2011

doi:10.5539/mer.v1n1p47

URL: http://dx.doi.org/10.5539/mer.v1n1p47

\begin{abstract}
We present here an extension of our finite element model of the Drosophila embryo to consider the interdependence of successive morphogenetic movements. A novel approach is used, which couples the Arbitrary Lagrangian Eulerian formulation with the harmonicparametrization. The combination of the two techniques allows to constantly update the deforming embryo geometry and simultaneously build an associated system of curvilinear coordinates. Thus, we are able to exactly describe the elementary cell deformations responsible for each biological event that are then defined with respect to the dynamic middle surface of the embryonic tissue and to their relative reference configuration. Both the active and the passive deformations occurring to the cells are considered through the deformation gradient decomposition. We develop a concurrent simulation of three morphogenetic movements: the ventral furrow invagination, the cephalic furrow formation and the germ band extension. The results show a consistent similarity with respect to the physical phenomena. More generally, the numerical approach that we propose could constitute a powerful tool to rigorously describe and simulate complex shape changes in biological systems.
\end{abstract}

Keywords: Shell-like cell deformations, Concurrent cell deformations, Drosophila embryo, Finite Element method, ALE method

\section{Introduction}

Biological tissues are heterogeneous materials whose constituents, and therefore structure, are continually changing due to growth and response of the tissue to its physical and chemical environment.

Multicellular organisms are fascinating in a double way. During the whole process of embryogenesis, cells play a major role at both the individual and the collective levels to guarantee the perfect synchronization necessary for a successful development. An error in this process may cause serious consequences for the adult animal. A multiscale approach is very appropriate in order to better understand the series of morphogenetic movements that determine the final shape of the embryo. Each one of these biological events stands up for specific elementary deformations involving groups of cells located at different regions of the system. Very often, some of these movements may occur simultaneously or successively, so that cells undergo more than one change in shape over the other. Therefore, cell strains that trigger later morphogenetic movements occur over a highly deformed shape created by previous movements, which causes a strong interdependence between all the events.

Among the large number of biological organisms often studied, Drosophila embryo, apart from its relevant molecular and genetics characteristics (Brouzés and Farge, 2004; Campos-Ortega and Hartenstein, 1985; Farge, 2003), is an interesting biomechanical system. In our previous paper (Allena et al., 2010a), we introduced a finite element model of it, which allowed us to simulate three individual movements occurring during the gastrulation 
phase of the development: the ventral furrow invagination (VFI), the cephalic furrow (CF) formation and the germ band extension (GBE). The three movements were independently described with respect to the same initial configuration without taking into account the chronology of the biological events. This was actually the main limitation of the work leading then to an only partially realistic simulation of the GBE, which in fact takes place after the furrows formation over a complete different shape of the embryo. To overcome this issue, we present here a major extension of our preceding model in order to describe each movement and its associated elementary deformations with respect to its specific and thus different initial configuration. We obtain then a concurrent simulation of the three morphogenetic movements.

Two advanced techniques are combined. Firstly, we use the Arbitrary Lagrangian-Eulerian (ALE) formulation to track the deforming biological tissue. In doing so, we constantly update the relative reference configuration with respect to which the shell-like cell deformations have to be described. Secondly, onto the ALE framework, we introduce the harmonicparametrization (Allena and Aubry, 2010b) to describe cell movements relative to the middle surface of the embryonic tissue. Similar developments have been proposed in literature by Marchandise (Marchandise et al., 2011) to properly and continuously define a deforming membrane middle surface and an off-distance variable. Besides, the authors use the term harmonic which we find now more appropriate than electric in our previous work (Allena and Aubry, 2010b). This novel approach allows building a special system of curvilinear coordinates to efficiently illustrate shell-like deformations of geometrically complex and three-dimensional thick membrane such as the Drosophila embryo. Furthemore, the approach may have a large range of applications in the domain of the computational system biology because it allows reproducing a series of consecutive and/or simultaneous cell shape changes within organisms with complex geometries.

The paper is divided into three main sections. In the first one, we provide a detailed description of the ALE formulation, of the harmonic parametrization of the Drosophila embryo and of the mechanics of the biological system. In the second one, we briefly describe the active deformations responsible for each morphogenetic movement that will be introduced into the finite element formulation through the decomposition of the deformation gradient. Finally, in the third section, the results of the work are presented.

\section{Kinematical and Mechanical Framework}

At the beginning of gastrulation, the geometry of the Drosophila embryo is simple and very similar to the one represented in Fig. 1.It has an approximated ellipsoidal shape with major axis $\mathrm{AB}$ equal to $500 \mu \mathrm{m}$, while the minor axes CE and DF are respectively $175 \mu \mathrm{m}$ and $165 \mu \mathrm{m}$ long (see Table 1). It is composed of a single layer of columnar epithelial cells (thickness $\mathrm{h}=15 \mu \mathrm{m}$ ), the blastoderm, which contains a slightly compressible fluid, the yolk, and it is surrounded by a semi-rigid shell, the vitelline membrane. The blastoderm forms a closed array of columnar cells having their apical surfaces facing outwards, the apico-basal axis is aligned along the axis of radial symmetry and each cell is in lateral contact with its neighbors (see Fig. 2 in Allena et al., 2010a).

\subsection{The arbitrary Lagrangian-Eulerian formulation}

In the embryo, cells cooperate together in order to guarantee the correct development of the organism. Therefore, very often the collective tissue deformations (macroscale) overcome the individual cells deformations (microscale). However, cells maintain their own orientation within the biological system. This means that at different regions of the embryo, the same strain may provide a complete different configuration. Thus, to be coherent regarding the biophysical phenomenon, cells shape changes have to be described with respect to a local and deforming coordinates system.

From a kinematical point of view, there are mostly three approaches to deal with such large deformations. In the Lagrangian or material description, every quantity is referenced only with respect to the initial configuration $\Omega_{0}$. In the Eulerian or spatial description, the unknowns are defined within the current configuration $\Omega_{\mathrm{t}}$. Although the two techniques are very useful, the first one does not allow to easily reproduce deformations occurring with reference to an intermediary configuration, while the second one does not enable to easily take into account deforming boundaries. However, in the Arbitrary Lagrange Eulerian (ALE) description (Donéa, 1983; Donéa and Huerta, 2003), a moving deformable reference configuration $\Omega_{\xi}$ is introduced so that the position $y$ of a particle initially identified by $\boldsymbol{p}$ is tracked at any time $t$ by the composition of the function $\boldsymbol{f}_{A L E}$

$$
\boldsymbol{y}=\boldsymbol{f}_{A L E}(\xi, t)
$$

and the moving reference $\xi$ is defined itself by its own kinematics as

$$
\xi=\phi(p, t)
$$


$f_{A L E}$ must obiouvsly follow the compatibility condition

$$
f_{A L E}(\phi(p, t), t)=f_{L}(p, t)
$$

with $f_{L}$ being the classical Lagrange description, which means that by composing the movement of the intermediate configuration with $\phi$ and $\boldsymbol{f}_{A L E}$, the material movement can be recovered. $\boldsymbol{f}_{A L E}$ will be computed from the mechanical equilibrium and the material response.

In order to determine the ALE placement, $\phi_{(p, t)}$ which is the only undefined function, we have chosen a very classical Laplace smoothing (Donéa, 1983). This means that $\phi_{(p, t)}$ must satisfy the following equation and boundary conditions

$$
\left\{\begin{array}{l}
\Delta \phi=0 \\
\phi_{\partial \Omega_{0}}=f_{L}
\end{array}\right.
$$

where $\partial \boldsymbol{\Omega}_{0}$ is the external boundary of the domain at the initial configuration. This equation is solved using the finite element method so that the nodes of the deformed mesh follow the $f_{L}$ placement on the boundaries and the Laplace smoothing inside. Obviously, this choice does not imply any particular assumptions regarding cell deformations, but it just provides an intermediary frame with respect to which further elementary cell strains may be described.

Now that we have built the framework to describe the embryo large deformations, we have to consider the fact that the elementary cell strains (i.e. elongation, shortening, bending...) are in fact typical shell-like local deformations. This means that we have to define a special curvilinear system of coordinates adapted to the biological system with an associated middle surface of the embryonic tissue, so that relative movements with respect to it may be described. In order to do this, we employ the harmonic technique that we have already presented in our previous work (Allena and Aubry, 2010b), but this time combined with the ALE formulation.

\subsection{The harmonic parametrization of the embryo geometry}

The elementary cell deformations observed during the gastrulation occur locally with respect to the deforming embryonic tissue, following a shell-like behavior. Thus, we need to build a specific curvilinear coordinates system adapted to the irregular geometry of the Drosophila embryo and formed by three parameters $\theta_{\xi}, \varphi_{\xi}$ and $\zeta_{\xi}$ which indeed will generalize spherical coordinates. Each one of them is computed using solutions of Laplace's equation on the given domain, with the appropriate boundary conditions and the proper geometry of the same domain (Allena and Aubry, 2010b).

Let be $\partial \boldsymbol{\Omega}_{\tilde{\xi} e}$ and $\partial \boldsymbol{\Omega}_{\xi i}$ respectively the outer and the inner surfaces of the embryo at any ALE relative intermediate configuration $\Omega_{\xi}$. If $\Delta$ is the Laplacian operator, then we define first the variable $\zeta_{\xi}$ within the thickness of the membrane through the Laplace's equation and Dirichlet's boundary conditions as follows

$$
\begin{cases}\Delta \zeta_{\xi}=0 & \text { inside } \Omega_{\xi} \\ \zeta_{\xi}=+\frac{h}{2} & \text { on the external boundary } \partial \Omega_{\xi e} \\ \zeta_{\xi}=-\frac{h}{2} & \text { on the internal boundary } \partial \Omega_{\xi i}\end{cases}
$$

where $h$ is the thickness of the embryonic tissue. As in our previous paper (Allena and Aubry, 2010b), $\zeta_{\xi}=0$ is assumed to be a good approximation of the middle surface $\sum_{\xi_{0}}$ (Fig. 2a), as long as the embryonic tissue is rather thin with respect to its largest diameter.

Then, from $\zeta_{\xi}$, an estimated normal vector $n_{0 \xi}$ to the shell middle surface $\zeta_{\xi}=0$ can be computed as follows (Fig. 3a)

$$
\boldsymbol{n}_{0 \xi}=\frac{\nabla \xi_{\xi}}{\left\|\nabla \zeta_{\xi}\right\|}
$$

Now, we need to define two other parameters, $\theta_{\xi}$ and $\varphi_{\xi}$, complementary to $\zeta_{\xi}$, which will serve both as surface 
coordinates. Unfortunately, the previous technique cannot be easily extended to the tangential boundaries, since the embryonic membrane is not a simply connected domain and it has only two boundaries. The clue is to introduce two slit surfaces across the thickness of the embryo (one for $\theta_{\xi}$ and one for $\varphi_{\xi}$ ) so that, following a loop inside the membrane, the two coordinates undergo a discontinuity when crossing their respective slit surfaces. $\theta_{\xi}$ is chosen to be the solution of the following Laplace's equation and boundary conditions, with [•] standing for the jump of the quantity across the slit

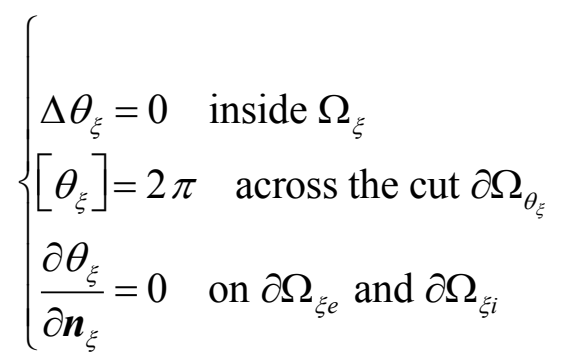

where $n_{\xi}$ is the geometrical normal vector and $\partial \boldsymbol{\Omega}_{\theta \xi}$ is the slit surface defined by $\{z=0, y<0\}$. To insure the uniqueness of $\theta_{\xi}$, its average value is also prescribed to vanish. The last parameter $\varphi_{\xi}$ can be similarly obtained through the slit surface $\partial \boldsymbol{\Omega}_{\varphi \xi}$ defined by $\{z=0, x>0\}$. In Fig. $2 \mathrm{~b}$ and $2 \mathrm{c}$, the isovalues of the two tangential parameters are represented. The associated vectors $\nabla \theta_{\xi}$ (Fig. 3b) and $\nabla \varphi_{\xi}$ (Fig. 3c), that complete the curvilinear system, are calculated as similarly as the normal vector $n_{0 \xi}$ (Eq. [6]). Obviously, for the real embryo this cooordinates system cannot be built by hand. Consequently, it is here computed using the same finite element mesh adopted to solve the mechanics of the system itself on the ALE configuration.

At this point, we have all the required kinematical tools to describe the particular mechanics of the biological system driven by the elementary cell deformations.

\subsection{Mechanics of the embryo}

Let us consider the sketch in Fig. 4, which represents the successive configurations of our framework. Let $\boldsymbol{p}$ and $\boldsymbol{x}$ be respectively the initial and the actual position of any material particle. Given the ALE formulation proposed above and the ALE position $\xi$ defined in Eq. [2], the ALE deformation gradient $\boldsymbol{f}_{A L E}$ is equal to

$$
\boldsymbol{F}_{A L E}=\boldsymbol{F} \cdot \boldsymbol{\Phi}^{-1}
$$

where

$$
\begin{gathered}
\boldsymbol{F}_{A L E_{-} i j}=\frac{\partial x_{i}}{\partial \xi_{j}} \\
\boldsymbol{F}_{i j}=\frac{\partial x_{i}}{\partial p_{j}} \\
\boldsymbol{\Phi}_{i j}=\frac{\partial \xi_{i}}{\partial p_{j}}
\end{gathered}
$$

The next step is to compute the stresses that develop inside the mechanical system. When the morphogenetic movements take place, we observe what can be called either the active or the individual deformation of the cells, which would be unconstrained if the cells were completely free. However, since the cells are in contact with one another and with the boundary components of the embryo (the yolk and the vitelline membrane), such deformations may lead to incompatibilities with respect to the continuity of the system, for instance overlapping or interstice formations. Thus, the passive deformation takes place and the cells react and rearrange themselves by an elastic response of the tissue. In order to consider both deformations, we use the deformation gradient decomposition (Smith, 1993; Rodriguez et al., 1994; Taber, 2007; Barret et al., 2007; Conte et al., 2007; Allena et al., 2010a). Alternative approaches based on active stresses have also been considered (Nobile et al., 2010). However, the deformation gradient decomposition employed here is simultaneously simpler, more robust and it 
has been extensively used for years in many other areas such as, for instance, crystal plasticity.

According to this method, the ALE deformation $\boldsymbol{f}_{A L E}$ of each particle of the system is then splitted into two parts: a non-stress generating deformation $\boldsymbol{F}_{a}$ (the active deformation) and a stress-generating deformation $\boldsymbol{F}_{m}$ (the passive deformation)

$$
\boldsymbol{F}_{A L E}=\boldsymbol{F}_{m} \boldsymbol{F}_{a}
$$

Let us define first the active deformation $\boldsymbol{F}_{\boldsymbol{a}}$.

Similarly to shell-like kinematics and using the full curvilinear coordinates system $\left(\theta_{\xi}, \varphi_{\xi}, \zeta_{\xi}\right)$ computed above by the harmonic parametrization (Sec. 2.2), let $\xi_{0 \xi}\left(\theta_{\xi}, \varphi_{\xi}\right.$, ) be a point on the middle surface of the embryo such that $\zeta_{\xi}=0$.. Then, we can define any point $\xi\left(\theta_{\xi}, \varphi_{\xi}, \zeta_{\xi}\right)$ through the thickness of the embryonic tissue at the relative reference configuration $\Omega_{\xi}$ as

$$
\xi\left(\theta_{\xi}, \varphi_{\xi}, \zeta_{\xi}\right)=\xi_{0 \xi}\left(\theta_{\xi}, \varphi_{\xi}\right)+\zeta_{\xi} n_{0 \xi}\left(\theta_{\xi}, \varphi_{\xi}\right)
$$

Thus, when the elementary active deformations take place (i.e. apical constriction, extension, shortening,...), any point $\boldsymbol{\xi}$ moves to an intermediate configuration $\overline{\boldsymbol{x}}$, different for each morphogenetic movement. Deriving $\overline{\boldsymbol{x}}$ will yield the expression of the active deformation gradient $\boldsymbol{F}_{\boldsymbol{a}}$, which is defined as follows (Smith, 1993) in the chosen curviliner system $\left(\theta_{\xi}, \varphi_{\xi}, \zeta_{\xi}\right)$.

$$
\boldsymbol{F}_{a}=\frac{\partial \overline{\boldsymbol{x}}}{\partial \theta_{\xi}} \otimes \nabla_{\xi} \theta_{\xi}+\frac{\partial \overline{\boldsymbol{x}}}{\partial \varphi_{\xi}} \otimes \nabla_{\xi} \varphi_{\xi}+\frac{\partial \overline{\boldsymbol{x}}}{\partial \xi_{\xi}} \otimes \nabla_{\xi} \zeta_{\xi}
$$

The covariant and the contravariant bases necessary to compute Eq. [12] are calculated as shown in (Allena and Aubry, 2010b).

Now, $\boldsymbol{F}_{a}$ being known, we can compute $\boldsymbol{F}_{m}$ from Eq. [10] and therefore the Second Piola-Kirchhoff tensor $\boldsymbol{S}_{\boldsymbol{m}}$ on the intermediary configuration $\bar{\Omega}$, which is obtained through the constitutive law of a Neo-Hookean material as follows (Allena, 2009; Ciarlet, 1988)

$$
\boldsymbol{S}_{m}=\alpha_{0} \boldsymbol{I}+\alpha_{1} \boldsymbol{E}_{m}+\alpha_{2} \boldsymbol{E}_{m}^{2}
$$

where $\alpha_{0}, \alpha_{1}, \alpha_{2}$ are the invariants of the Green-Lagrange strain tensor $E_{m}=\frac{1}{2}\left(C_{m}-I\right)$, with $\mathrm{C}_{\mathrm{m}}=F_{m}^{T} F_{m}=$ $F_{a}^{-T} F^{T} F F_{a}$, measures the elastic passive deformation. TheYoung's modulus $E$ and Poisson's ratio $v$ have been chosen equal to $100 \mathrm{~Pa}$ (Wiebe and Brodland, 2005) and 0.45 respectively (at the limit of small strains).By using a purely elastic model we are disregarding any viscous dissipative phenomenon. We are aware that the presence of cytoplasm or potential remodeling processes within the cell may require more realistic visco-elastic models, and therefore we are inducing some errors in the resulting stresses of our model. However, from the ablation experiments shown in (Supatto et al., 2005), it can be concluded that the elastic deformations cannot be disregarded. Furthermore, the aim of the present work is to analyze the plausibility of the interdependence of successive morphogenetic movements which constitutes a fundamental aspect during embryogenesis, regardless of the values of the stresses. Since no measurements of the stress field during Drosophila embryo development have been reported so far, the inclusion of more sophisticated constitutive models would require guessing some of their material parameters. We have instead decided to resort to simpler models, but using material parameters that can be experimentally estimated.

To compute the weak form of the system, we need to calculate the First Piola-Kirchhoff tensor $\boldsymbol{\pi}_{\xi}$ with respect to the ALE configuration $\Omega_{\xi}$ as follows

$$
\boldsymbol{\pi}_{\xi}=J_{a} \boldsymbol{S}_{m} \boldsymbol{F}_{a}^{-T}
$$

where $J_{a}=\operatorname{Det}\left[F_{a}\right]$. Consequently, by the constitutive equation (Eq. [13]), $\boldsymbol{\pi}_{\xi}$ is a coupled function of $\boldsymbol{F}_{a}$ and $\boldsymbol{F}_{A L E}$. Finally, the weak form reads as

$$
\int_{\Omega_{\xi}} \operatorname{Tr}\left(\boldsymbol{\pi}_{\xi} \boldsymbol{D}_{\xi} \boldsymbol{w}_{\xi}^{T}\right) d V=\int_{\partial \Omega_{\xi}}\left(\boldsymbol{w}_{\xi}, f_{s} J_{\xi} \boldsymbol{F}_{\xi}^{-T}\left(\boldsymbol{n}_{\xi}\right)\right) d S
$$


where $(a, b)$ is the dot product between two vectors $\boldsymbol{a}$ and $\boldsymbol{b}$ and $\left(D_{\xi} w_{\xi}\right)_{m n}=\frac{\partial w_{\xi, m}}{\partial \xi_{n}}$.

$\boldsymbol{f}_{s}$ indicates the surface forces, $\boldsymbol{w}_{\xi}$ is any displacement test function and $J_{\xi}=\operatorname{Det}\left[F_{\xi}\right]$. It has to be remembered that simultaneously to the mechanical weak form described above, we also solve the weak forms relative to the three parameters $\left(\theta_{\xi}, \varphi_{\xi}, \zeta_{\xi}\right)$ and to the ALE position $\phi_{\xi}$ as follows

$$
\begin{aligned}
& \left\{\begin{array}{l}
\int_{\Omega_{\xi}}\left(\nabla \theta_{\xi}, \nabla \hat{\theta}_{\xi}\right) d V=0 \\
{\left[\begin{array}{l}
\left.\theta_{\xi}\right]=2 \pi
\end{array}\right.}
\end{array}\right. \\
& \left\{\begin{array}{l}
\int_{\Omega_{\xi}}\left(\nabla \varphi_{\xi}, \nabla \hat{\varphi}_{\xi}\right) d V=0 \\
{\left[\begin{array}{l}
\varphi_{\xi} \\
\varphi_{\xi}
\end{array}=2 \pi\right.}
\end{array}\right. \\
& \left\{\begin{array}{l}
\int_{\Omega_{\xi}}\left(\nabla \zeta_{\xi}, \nabla \hat{\zeta}_{\xi}\right) d V=0 \\
\zeta_{\xi}= \pm \frac{h}{2} \text { on } \partial \Omega_{\xi i} \text { and } \partial \Omega_{\xi e}
\end{array}\right. \\
& \left\{\begin{array}{l}
\int_{\Omega_{\xi}}\left(\nabla \phi_{\xi}, \nabla \hat{\phi}_{\xi}\right. \\
\phi_{\xi}=f_{L}
\end{array}\right) d V=0
\end{aligned}
$$

Where $\hat{\theta}_{\xi} \widehat{\varphi}_{\xi}$, and $\widehat{\phi}_{\xi}$ are the test functions associated with each parameter. This tightly non-linear coupled problem is computed at each step of the parameter $t$ with the following staggered scheme:

(1) from the current updated geometry $\Omega_{\xi}$ compute the parameters $\theta_{\xi}, \varphi_{\xi}$ and $\zeta_{\xi}$,

(2) compute the active deformations from the curvilinear frame;

(3) compute the displacements of the embryonic tissue;

(4) eventually re-update the current geometry through the ALE weak form.

Even though they are not geometrically modeled here, the vitelline membrane and the internal yolk and their relative boundary conditions are taken into account during step 3, as similarly as for our previous work (Allena et al., 2010a), respectively by contact and global volume boundary conditions.

In the previous sections, the three main ingredients of our work (the ALE formulation (Sec. 2.1), the harmonicparametrization (Sec. 2.2) and the deformation gradient method) have been presented and we have shown how they interplay. In the next part of the manuscript, we detail the active cell deformations responsible for each morphogenetic movement and we deduce the associated active deformation gradients $\boldsymbol{F}_{a}$.

\section{Morphogenetic Movements}

We focus here on the concurrent simulation of three morphogenetic movements: the VFI, the CF formation and the GBE. The first two take place simultaneously, while the GBE starts almost at the end of the VFI. The active deformations responsible for the VFI and the CF occur thus on the undeformed embryo, while those responsible for the GBE involve cells that have already undergone significant changes in shape. The concurrent simulation can then be divided into two phases: the first one including the VFI and the CF formation and the second one including the GBE. In the next paragraphs, we briefly describe the biological events and the associated cellular movements that are necessary to analytically express the intermediary position $\bar{x}$ (Sec. 2.3) and therefore the active deformation gradient $\boldsymbol{F}_{a}$ by derivation.

\subsection{First phase: the ventral and the cephalic furrow}

The VFI is one of the most studied morphogenetic movements occurring during Drosophila gastrulation. Several numerical models have been proposed in literature during the last decades (Alberch et al., 1981; Clausi and Brodland, 1994; Davidson et al., 1995; Pouille and Farge, 2007; Barret et al., 2007; Ramasubramamian and Taber, 2008; Conte et al., 2007; Allena et al., 2010a). All these works, whether they are two or three dimensional, 
only focus on the individual simulation of the VFI per se. Here however, we correctly simulate it as a required stage for further movements such as the GBE.

As we have amply discussed in our previous work (Allena et al., 2010a), many experimental observations and studies show that the VFI is triggered by a series of changes in shapeof individual cells in the areas of active movements (Alberch et al., 1981; Brouzés and Farge, 2004; Costa et al., 1993; Leptin and Grunewald, 1990; Leptin, 1999; Sweeton et al., 1991). Practically, the cells change their shape from a cylindrical to a more trapezoidal form, inducing the curvature necessary for the invagination of the mesoderm into the yolk. Accordingly to this and to our previous results (Allena et al., 2010a), here we consider the apical constriction along $\theta_{\xi}$ as the only responsible of morphogenetic movement.

Thus, we can analytically translate such a bending mode and write the intermediate position $\bar{x}^{V F I}\left(\theta_{\xi}, \varphi_{\xi}, \zeta_{\xi}\right)$ in the specific curvilinear coordinates system as follows

$$
\overline{\boldsymbol{x}}^{V F I}=\xi_{0 \xi}\left(\bar{\theta}_{\xi}, \varphi_{\xi}\right)+\zeta_{\xi} n_{0 \xi}\left(\bar{\theta}_{\xi}, \varphi_{\xi}\right)
$$

with

$$
\bar{\theta}_{\xi}=\theta_{\xi}+\alpha^{V F I}(t) \frac{2 \zeta_{\xi}}{h} m\left(\theta_{\xi}\right)
$$

Where $\alpha^{V F I}(t)=\alpha_{V F I} \cdot t$ is the amplitude of the active deformation, with $\alpha_{V F I}$ a constant and $t$ the evolution parameter. $m\left(\theta_{\xi}\right)$ is a periodic function that mimics the cell boundaries by appropriately modulating the intensity of the active deformation (see (Allena et al., 2010a) for an explicit expression). The active deformation is introduced in a restrained region of the embryo, the active region, where therefore $\alpha_{V F F} \neq 0$. In the specific case of the VFI, the active region covers almost $70 \%$ of the ventral embryo as it is observed in reality (Fig. 5). A characteristic function (Allena and Aubry, 2010c) built from a smooth and regularized Heaviside function is used to define the region.

The CF formation takes place simultaneously with the VFI and it is regulated by the same elementary cell deformations (Allena et al., 2010a; Allena et al., 2010c; Vincent et al., 1997). Nevertheless, two main differences can be noticed. First, this time the apical constriction of the cells occurs along the anterior-posterior axis rather than along the section of the embryo. Second, the invagination is smaller than for the ventral furrow, thus a smaller number of cells is involved in the process.

To reproduce the movement, we consider again the apical constriction the unique active deformation in control of the movement (Allena et al., 2010c), but this time it takes place along $\varphi_{\xi}$.

Therefore, the intermediate position $\bar{x}^{C F}\left(\theta_{\xi}, \varphi_{\xi}, \zeta_{\xi}\right)$ can be written as

$$
\overline{\boldsymbol{x}}^{C F}=\boldsymbol{\xi}_{0 \xi}\left(\theta_{\xi}, \bar{\varphi}_{\xi}\right)+\zeta_{\xi} \boldsymbol{n}_{0 \xi}\left(\theta_{\xi}, \bar{\varphi}_{\xi}\right)
$$

where

$$
\bar{\varphi}_{\xi}=\varphi_{\xi}+\alpha^{C F}(t) \frac{2 \zeta_{\xi}}{h} m\left(\varphi_{\xi}\right)
$$

with $\alpha^{C F}(t)=\alpha_{C F} \cdot t$, with $\alpha_{C F}$ a constant, and $m\left(\varphi_{\xi}\right)$ is again a periodic function (Allena and Aubry, 2010c). In order to reproduce the movement of the furrow towards the anterior pole, we use, as in our previous work (Allena and Aubry, 2010c), a dynamic active region which allows to take into account the rotation of the cells with respect to the vertical axis of the embryo. At the initial configuration $\Omega_{0}$, the active region appears as in Fig. 5 .

The active deformation gradient that combines the two concurrent morphogenetic movements is then equal to the composition of the two individual active deformation gradients, $\boldsymbol{F}_{\boldsymbol{a}}{ }^{\boldsymbol{V} \boldsymbol{F}}$ and $\boldsymbol{F}_{\boldsymbol{a}} \boldsymbol{C F}$, that are obtained according to Eq. [12].

Once the two furrows have formed, the germ band starts to extend. At this stage of the embryogenesis, the embryo has a completely different geometry (Fig. 6) from the initial one that we have previously considered for 
the VFI and the CF formation (Fig. 1). Then, this is the relative reference configuration with respect to which the elementary deformations responsible for the GBE have to be described.

\subsection{Second phase: the GBE}

The germ band, located at the ventral region of the embryo, starts to extend almost at the end of the ventral furrow formation. The elongation of the tissues is due to a convergent-extension movement of a population of cells at the centro-lateral region of the embryo that intercalate and interpose themselves between their dorsal or ventral neighbors(Weliky and Oster, 1990).This results in a decrease of the number of cells along the dorsal-ventral axis and in an increase of the number of cells along the anterior-posterior axis (Keller et al., 2000). As for the previous work (Allena et al., 2010a), we do not simulate the intercalation at the cellular scale, since the cells are not here geometrically modeled. We propose a uniform movement of compression-extension tangential to the middle surface of the embryonic tissue. Such a movement is described with respect to the relative reference configuration $\Omega_{\xi}$ at the end of the formation of the two furrows (cephalic and ventral) (Fig. 6) that has been tracked by the ALE formulation. Furthermore, the system of curvilinear coordinates $\left(\theta_{\xi}, \varphi_{\xi}, \zeta_{\xi}\right)$ has been updated as shown in Fig. 7together with the three associated vectors (Fig. 8).

Thus, the intermediate position $\bar{x}^{G B E}\left(\theta_{\xi}, \varphi_{\xi}, \zeta_{\xi}\right)$ is equal this time to

$$
\overline{\boldsymbol{x}}^{G B E}=\boldsymbol{\xi}_{0 \xi}\left(\bar{\theta}_{\xi}, \bar{\varphi}_{\xi}\right)+\zeta_{\xi} n_{0 \xi}\left(\bar{\theta}_{\xi}, \bar{\varphi}_{\xi}\right)
$$

where

$$
\begin{gathered}
\bar{\theta}_{\xi}=\left(1+\alpha_{\theta_{\xi}}^{G B E}(t)\right) \theta_{\xi} \\
\bar{\varphi}_{\xi}=\left(1+\varphi_{\xi}\right)\left(1+\alpha_{\varphi_{\xi}}^{G B E}(t)\right) \varphi_{\xi}
\end{gathered}
$$

With $\alpha_{\theta_{\xi}}^{G B E}(t)=\alpha_{G B E_{-} \theta \xi} \cdot t$ and $\alpha_{\varphi_{\xi}}^{G B E}(t)=\alpha_{G B E_{-} \varphi \xi^{\xi}} \cdot t$ the amplitudes, respectively, of the shortening from the dorsal to the ventral region of the embryo and of the lengthening along the anterior-posterior axis $\left(\alpha_{G B E_{-} \theta \xi^{\prime}}\right.$ and $\alpha_{G B E_{-} \varphi \xi^{*}}$ are two constants). The active region where the active deformations take place is represented in red in Fig 9.As for the previous movements, $\boldsymbol{F}_{\boldsymbol{a}}{ }^{\boldsymbol{G} B E}$ is computed according to Eq. [12].

\section{Results}

The present model and the special weak forms associated to it have been developed using the finite element program COMSOL Multiphysics (v. 3.5a, Comsol Inc.).

To discuss the results of the simulation, we propose a set of pictures that show the embryo from different view-points: from the rear (Fig. 10), from the bottom (Fig. 11a :e) and cross sections (Fig. 11f:1), from the front (Fig. 12). As we can observe from Fig. 11a and 12a, the three active regions intersect at different zones of the embryo and such overlap lasts all along the simulation. In our opinion, this should not be an issue, except for the presence of further strains occurring to the tissue and in particular at the intersection region between the VFI and the $\mathrm{CF}$ where an additional invagination takes place. The formation of the two furrows is clearly evident during the first phase of the simulation, from $t=0$ to $t=0.012$ (Fig. 10a :c, 11a :c, 12a :c). Also, the rotation of the cephalic furrow with respect to the vertical axis of the embryo is evident. According to Eq. [21] and [23], we have calculated the maximal active deformations which would occur to the cells if they were completely free. We found a maximal apical constriction along $\varphi_{\xi}$ of $4.5 \mu \mathrm{m}$ for the $\mathrm{CF}$ and a maximal apical constriction along $\theta_{\xi}$ of $7.2 \mu \mathrm{m}$ for the VFI. These values are of the order of magnitude of the individual simulations shown in our previous work (Allena et al., 2010a) and they lead to invaginations very similar to the ones experimentally observed (see Fig.1 and 3 in (Allena et al., 2010a)). The second phase, corresponding to the extension of the GBE, runs from $t=0.012$ to $t=0.214$. The lengthening of the tissue towards the posterior pole of the embryo is distinctly visible in Fig. 10d,e; 12d,e. With respect to the individual simulation of the GBE proposed in (Allena et al., 2010a), the movement is more pronounced and therefore more similar to the real morphogenetic movement. The extension is not symmetric anymore (Allena et al., 2010a) given the gradient of the deformation introduced above (Eq. [26]). Therefore, there is an elongation of about $35 \mu \mathrm{m}$ towards the posterior pole and of about $15 \mu \mathrm{m}$ towards the anterior pole. Even though the posterior extension is still inhibited by the sharpened posterior pole, it is found that the tissue tends to overtake the posterior pole (Fig. 12e). The next step would be to 
push forward the posterior extension in order to be able to observe the series of small invaginations on the dorsal part of the embryo, which seem to start here (Fig. 12e to compare with Fig. 3 in (Allena et al., 2010a)). Nevertheless, the present results match much better than the ones for the individual simulation (Allena et al., 2010a) when compared to experimental images. This certainly prooves the main advantage of the novel approach introduced here that allows to precisely describe the convergent-extension movement responsible for the GBE with respect to the specific reference configuration produced by the VFI and the CF formation.

As in our previous work (Allena et al., 2010a), we estimate the induced pressures within the tissue in the non-active region of the embryo during the simulation. In particular, we want to evaluate the pressure variation during the GBE in order to compare such stress to the ones experimentally observed that seem to be responsible of a mechanotransduction phenomenon at the anterior pole of the embryo. At $t=0.012 \mathrm{we}$ find that the pressures in the non-active region are between $-313 \mathrm{~Pa}$ and $146 \mathrm{~Pa}$ and at the anterior and the posterior poles we have respectively $0.14 \mathrm{~Pa}$ and $0.21 \mathrm{~Pa}$. At $t=0.226$, at the end of the second phase, the induced pressures are between $-133 \mathrm{~Pa}$ and $289 \mathrm{~Pa}$, while at the anterior and the posterior pole the pressure is equal respectively to $-2.33 \mathrm{~Pa}$ and 1.33Pa. These last values are in the order of the ones found in our previous work (Allena et al., 2010a). Furthermore, we oberve a compression of the cells at the anterior pole $(-2.33 \mathrm{~Pa})$ which confirms the conclusions pointed out by Supatto (Supatto et al., 2005).

The stress state at the end of the VFI could be the main cause of the cell shape changes triggering the early first phase of the GBE, as pointed out by Butler and co-workers (Butler et al., 2009). Actually, in support of a direct role for the invaginating mesoderm, they found that the germ band extends faster closest to the midline during the first $20 \mathrm{mn}$ of the biological event, suggesting an axial pull. Therefore, they suggest a possible mechanism by which the mesoderm, once invaginated, undergoes convergence and extension, elongating the tube along the antero-posterior axis of the embryo. The convergence-extension could drag the midline cells and the adjacent tissue through an anterior-posterior tensile force. In order to find some evidence of this mechanical phenomenon, we have calculated the normal stress $\sigma_{\varphi_{\xi} \varphi_{\xi}}$ to the embryonic tissue at the end of the first phase of our simulation (once both the VFI and CF have completely formed). To do that, we compute the Cauchy stress according to Fig. 4 and the following equation

$$
\boldsymbol{\sigma}=\frac{1}{J_{m}} \boldsymbol{F}_{m} \boldsymbol{S}_{m} \boldsymbol{F}_{m}^{T}
$$

where $J_{m}$ is the determinant of the passive deformation gradient $F_{m}$ and $S_{m}$ is the associated second Piola-Kirchhoff stress. Then, $\sigma_{\varphi_{\xi} \varphi_{\xi}}$ is obtained by projecting $\sigma$ along the tangential vector to the embryonic membrane $\nabla \varphi_{\xi}$ as follows

$$
\sigma_{\varphi_{\xi} \varphi_{\xi}}=\left(\sigma\left(\nabla \varphi_{\xi}\right), \nabla \varphi_{\xi}\right)
$$

The computation of $\sigma_{\varphi_{\xi} \varphi_{\xi}}$ is possible thanks to the special parametrization of the embryonic system. This confirms the efficiency and the adaptability of the proposed technique.

From Fig. 13, we notice that $\sigma_{\varphi_{\xi} \varphi_{\xi}}$ is positive over the first phase of the concurrent simulation $(0<t<0.012)$ within the active region of the VFI, which means that a tensile force occurs (Butler et al., 2009). At $t=0.012$, $\sigma_{\varphi_{\xi} \varphi_{\xi}}$ varies between $33 \mathrm{~Pa}$ and $125 \mathrm{~Pa}$ and it is greater at the left end side of the region. If the stress state that we find corresponds to the real one, we can confirm the hypothesis pointed out by Butler (Butler et al., 2009), according to which cell shape changes during the early fast phase of the GBE is a passive response to an antero-posterior force occurring during the VFI.

\section{Conclusions}

We have presented a major extension of our numerical model of the Drosophila embryo to take into account the interdependence of successive morphogenetic movements, which constitutes a fundamental aspect in embryogenesis. We have developed a concurrent simulation of three morphogenetic movements during the early gastrulation phase of the development: the ventral furrow invagination, the cephalic furrow formation and the germ band extension. The main novelty of the approach used here lays in coupling the ALE formulation and the harmonicparametrization of the embryo geometry. The first technique allows us to constantly update the deforming geometry of the embryo and therefore the correlated finite elements mesh. The second technique 
enables to build the associated system of curvilinear coordinates at each step of the simulation. Doing so, we are able to exactly describe the shell-like cell deformations responsible for each morphogenetic movement with respect to the moving reference configuration. Additionally, we take into account the fact that same populations of cells may undergo successive active strains during embryogenesis.

Here, we show that the three biological events correctly occur. In particular, the germ band extension simulation is much more realistic than in our previous work (Allena et al., 2010a) thanks to the precise description of the elementary cell deformation responsible for it. Therefore, we are convinced that our work could be very useful to confirm, even qualitatively, specific hypotheses according to which early morphogenetic movements may be the direct cause of later ones (Butler et al., 2009). This type of scenario would suggest then a straight interdependence between biological events, an aspect which has been so far little considered.

Even though the numerical approach has been here applied to the specific case of the Drosophila embryo, it could be adopted and adapted to rigorouslymodel any other type of concurrent or successive cell deformations within complex and dynamic multicellular organisms.

\section{References}

Alberch O. et al. (1981). The mechanical basis of morphogenesis. Epithelial folding and invagination. Dev. Biology, 75, 446-462.

Allena R. (2009). Numerical simulation of morphogenetic movements in Drosophila embryo. PhD Thesis, http://hal.archives-ouvertes.fr/tel-00429691/

Allena R., Mouronval A.-S., \& Aubry D. (2010a). Simulation of multiple morphogenetic movements in Drosophila embryo by a single 3D Finite Elements model. Journal of the Mechanical Behavior of Biomedical Materials. http://dx.doi.org/10.1016/j.jmbbm.2010.01.001

Allena R., \& Aubry D. (2010b). A novel technique to parametrize shell-like deformations inside biological membranes. Computational Mechanics, http://dx.doi.org/10.1007/s00466-010-0551-8

Allena R., \& Aubry D. (2010c). An extensive numerical simulation of cephalic furrow formation in Drosophila embryo. Computer methods in biomechanics and biomedical engineering. http://dx.doi.org/10.1080/10255842.2010.539564

Barret K. et al. (2007). A deformation gradient decomposition method for the analysis of the mechanics of morphogenesis. Journal of Biomechanics, 40, 1372-1380. http://dx.doi.org/10.1016/j.jbiomech.2006.05.006

Brouzés E., \& Farge E. (2004). Interplay of mechanical deformation and patterned gene expression in developing embryo. Curr. Opin. Genet. Dev., 14, 367-74. http://dx.doi.org/10.1016/j.gde.2004.06.005

Butler L. C. et al. (2009). Cell shape changes indicate a role for extrinsic tensile forces in Drosophila germ-band extension. Nature Cell Biology. http://dx.doi.org/10.1038/ncb1894

Campos-Ortega, J. A., \& Hartenstein, V. (1985). The Embryonic development of Drosophila melanogaster. Springer-Verlag, Berlin.

Ciarlet P. G. (1988). Mathematical elasticity, volume I: Three-dimensional elasticity. Acta Applicandae Mathematicae, 18(2), 190-195.

Clausi D. A., \& Brodland G. W. (1994). Embryonic tissue morphogenesis modeled by FEM. J. Biomech. Engin., 116, 146-156. http://dx.doi.org/10.1115/1.2895713

Conte V. et al. (2007). A 3D finite element model of ventral furrow invagination in the Drosophila Melanogaster embryo. J. of the mechanical behavior of biomedical materials, 1, 188-198. http://dx.doi.org/10.1016/j.jmbbm.2007.10.002

Costa M. et al. (1993). Gastrulation in Drosophila: cellular mechanisms of morphogenetic movements in: Bate M., Martinez-Arias A. (eds), The Development of Drosophila. Cold Spring Harbor Laboratory Press, New York, pp. $425-466$.

Davidson L. et al. (1995). How do sea urchins invaginate? Using biomechanics to distinguish between mechanisms of primary invagination. Development, 121, 2005-2018.

Donéa J. (1983). Arbitrary Lagrangian-Eulerian finite element methods, in: T. Belytschko, T.J.R. Hughes (Eds.), Computational Methods for Transient Analysis, Elsevier, pp. 474-516 (Chapter 10).

Donéa J., \& Huerta A. (2003). Finite element method for flow problems. Wiley \& Sons, England.

Farge E. (2003). Mechanical induction of twist in the Drosophila foregut/stomodeal primordium. Current Biology, 


\section{3, 1365-1377. http://dx.doi.org/10.1016/S0960-9822(03)00576-1}

Keller R. et al. (2000). Mechanisms of convergence and extension by cell intercalation. Philos. Trans. R. Soc. Lond. B. Biol. Sci., 355(1399), 897-922. http://dx.doi.org/10.1098/rstb.2000.0626

Leptin M. \& Grunewald B. (1990). Cell shape changes during gastrulation in Drosophila. Development, 110: 73-84.

Leptin M. (1995). Drosophila gastrulation: from pattern formation to morphogenesis. Annu. Rev. Cell Dev., 11: 189-212. http://dx.doi.org/10.1146/annurev.cb.11.110195.001201

Leptin M. (1999). Gastrulation in Drosophila: the logic and the cellular mechanisms. EMBO J, 18: 3187-3192. http://dx.doi.org/10.1093/emboj/18.12.3187

Marchandise E., Carton de Wiart C., Vos W.G., Geuzaine C., \& Remacle J.-F. (2011). High-quality remeshing using harmonic maps Part II: Surfaces with high genus and of large aspect ratio. IJNME. http://dx.doi.org/10.1002/nme.3099

Nobile F., Quarteroni A., \& Ruiz-Baier R. (2010). Numerical solution of an active strain formulation for the electromechanical activity in the heart. MOX-Report No. 20/2010.

Pouille P. A., Farge E. (2007). Hydrodynamic simulation of multicellular embryo invagination. Phys. Biol., $5 / 015005$.

Ramasubramamian A. \& Taber L. A. (2008). Computational modeling of morphogenesis regulated by mechanical feedback. Biomechan. Model. Mechanobiol., 7, 77-91. http://dx.doi.org/10.1007/s10237-007-0077-y

Rodriguez EK, Hoger A, and McCulloch AD. (1994). Stress-dependent finite growth in soft elastic tissues. J.Biomechanics, 27, 455-46. http://dx.doi.org/10.1016/0021-9290(94)90021-3

Smith D. R. (1993). An introduction to continuum mechanics. Kluwer Academic Publishers, The Netherlands.

Supatto W. et al. (2005). In vivo modulation of morphogenetic movements in Drosophila embryos with femtosecond laser pulses. PNAS, 102, 1047-1052. http://dx.doi.org/10.1073/pnas.0405316102

Sweeton D. et al. (1991). Gastrulation in Drosophila: the formation of the ventral furrow and posterior midgut invaginations. Development, 112, 775-789.

Taber L. A. (2007). Theoretical study of Beloussov's hyper-restoration hypothesis for mechanical regulation of morphogenesis. Biomechan. Model. Mechanobiol., 7(6), 727-41.

Vincent A., Blankenship J. T., \& Wieshaus E. (1997). Integration of the head and trunk segmentation systems controls cephalic furrow formation in Drosophila. Development, 124, 3747-3754.

Weliky M., \& Oster G. (1990). The mechanical basis of cell rearrangement. Development, 106, 372-386.

Wiebe C. \& Brodland G. W. (2005). Tensile properties of embryonic epithelia measured using a novel instrument. J. Biomech., 38, 2087-2094. http://dx.doi.org/10.1016/j.jbiomech.2004.09.005 
Table 1. Principal variables of the model.

\begin{tabular}{|c|c|c|}
\hline Variable & Notation & Value \\
\hline Ellipsoid major axis & $\mathrm{AB}$ & $500 \mu m$ \\
\hline Ellipsoid minor axis & $\mathrm{CE}$ & $175 \mu m$ \\
\hline Ellipsoid minor axis & $\mathrm{DF}$ & $165 \mu \mathrm{m}$ \\
\hline Ellipsoid thickness & $\mathrm{h}$ & $15 \mu m$ \\
\hline Young's modulus & $\mathrm{E}$ & $100 \mathrm{~Pa}$ \\
\hline Poisson's ratio & $v$ & 0.45 \\
\hline Maximal active apical constriction along $\varphi_{\xi}(\mathrm{CF})$ & - & $4.5 \mu m$ \\
\hline Maximal active apical constriction along $\theta_{\xi}(\mathrm{VFI})$ & - & $7.2 \mu m$ \\
\hline Extension towards PP (GBE) & - & $35 \mu m$ \\
\hline Extension towards AP (GBE) & - & $15 \mu m$ \\
\hline Pressure at $\mathrm{t}=0.012$ in the non active region & $\mathrm{p}_{1 \mathrm{st}}$ & $-313 \mathrm{~Pa}<\mathrm{p}_{1 \mathrm{st}}<146 \mathrm{~Pa}$ \\
\hline Pressure at $\mathrm{t}=0.012$ at the anterior pole & $\mathrm{p}_{1 \mathrm{st} \_\mathrm{AP}}$ & $0.14 \mathrm{~Pa}$ \\
\hline Pressure at $\mathrm{t}=0.012$ at the posterior pole & $\mathrm{p}_{1 \mathrm{st} \_\mathrm{PP}}$ & $0.21 \mathrm{~Pa}$ \\
\hline Pressure at $\mathrm{t}=0.226$ in the non active region & $\mathrm{p}_{2 \mathrm{nd}}$ & $-133 \mathrm{~Pa}<\mathrm{p}_{2 \mathrm{nd}}<289 \mathrm{~Pa}$ \\
\hline Pressure at $\mathrm{t}=0.226$ at the anterior pole (at $\mathrm{t}=0.012$ ) & $\mathrm{p}_{2 \text { nd_AP }}$ & $-2.33 \mathrm{~Pa}$ \\
\hline Pressure at $\mathrm{t}=0.226$ at the posteriorr pole (at $\mathrm{t}=0.012$ ) & $\mathrm{p}_{2 \mathrm{nd} / \mathrm{PP}}$ & $1.33 \mathrm{~Pa}$ \\
\hline Normal stress & $\sigma_{\varphi_{\xi} \varphi_{\xi}}$ & $33 \mathrm{~Pa}<\sigma_{\varphi_{\xi} \varphi_{\xi}}<125 \mathrm{~Pa}$ \\
\hline
\end{tabular}




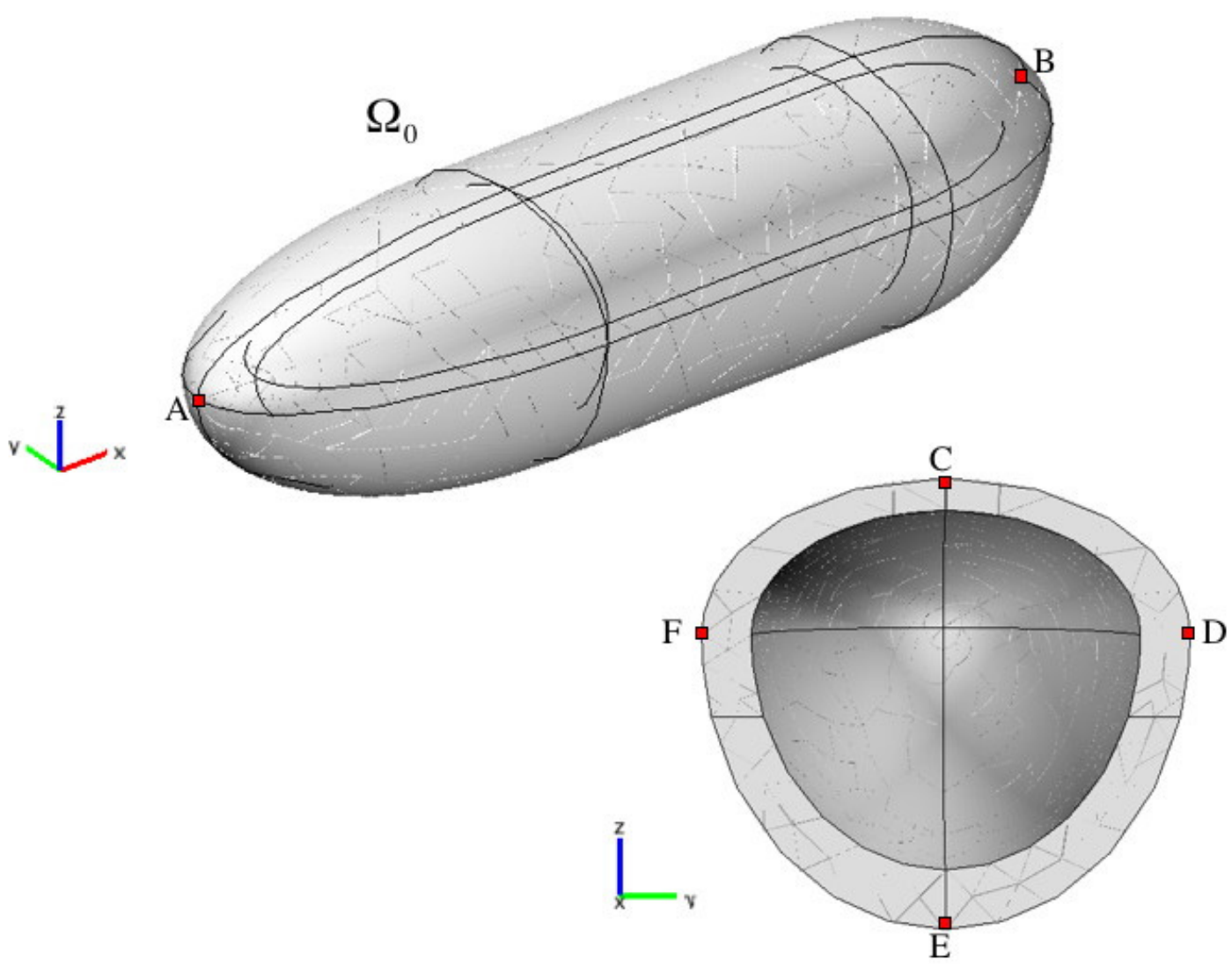

Figure 1. The geometry of the Drosophila embryo, which has been built from an anterior and an exterior ellipsoid retrieved from real embryo images. The major axis $\mathrm{AB}$ is $500 \mu \mathrm{m}$, while the cross axes $\mathrm{CE}$ and $\mathrm{DF}$ are respectively $175 \mu \mathrm{m}$ and $165 \mu \mathrm{m}$. The thickness of the embryo $h$ is equal to $15 \mu \mathrm{m}$ 


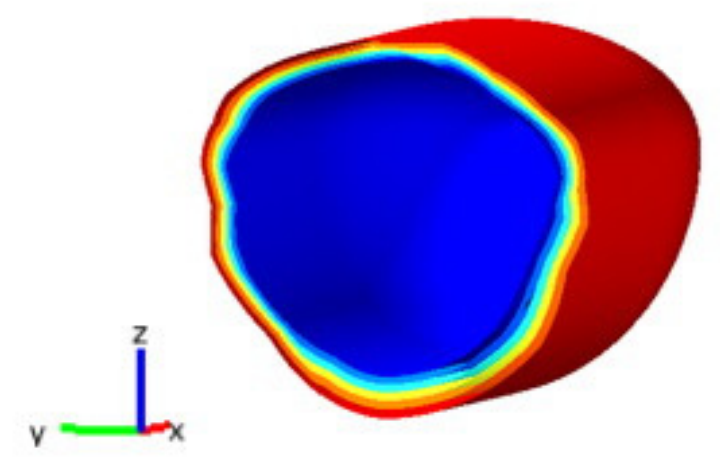

(a)

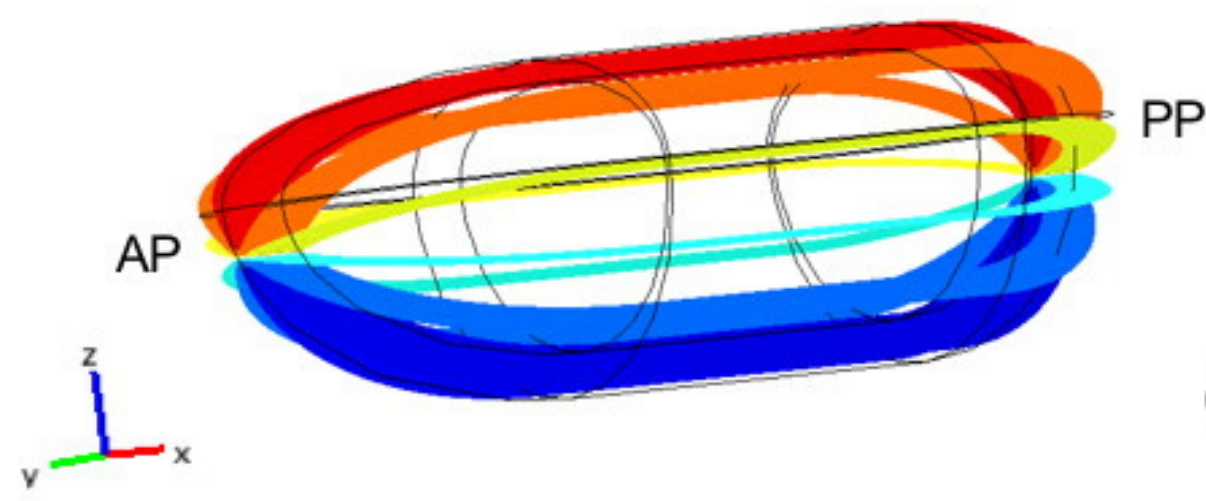

(b)

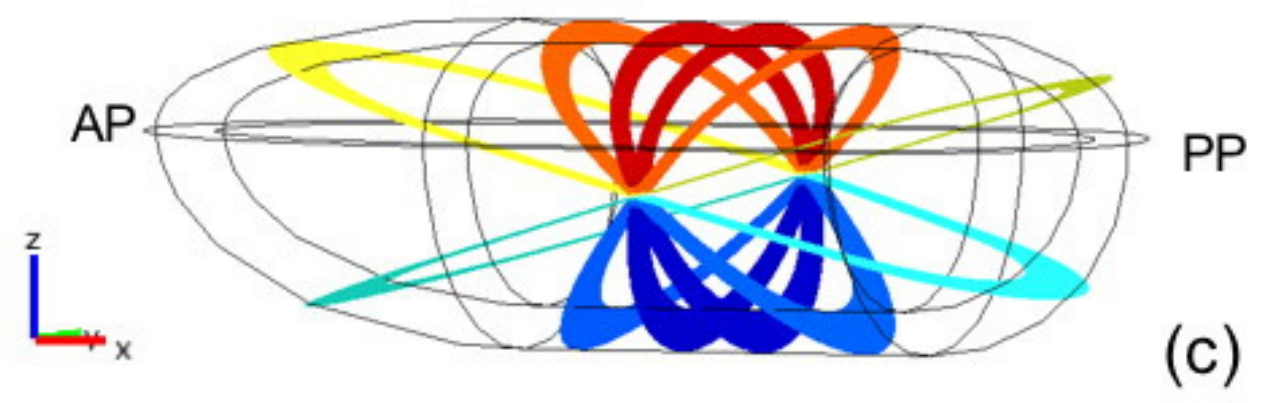

Figure 2. The isovalues of the threeparameters obtained by the harmonic parametrization of the geometry at the initial configuration $\Omega_{0}: \zeta_{\xi}(\mathrm{a}), \theta_{\xi}(\mathrm{b})$ and $\varphi_{\xi}(\mathrm{c})(\mathrm{AP}=$ anterior pole, $\mathrm{PP}=$ posterior pole $)$ 


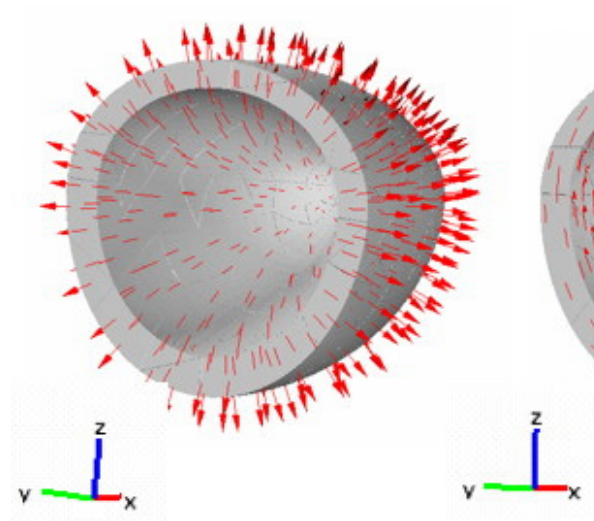

(a)

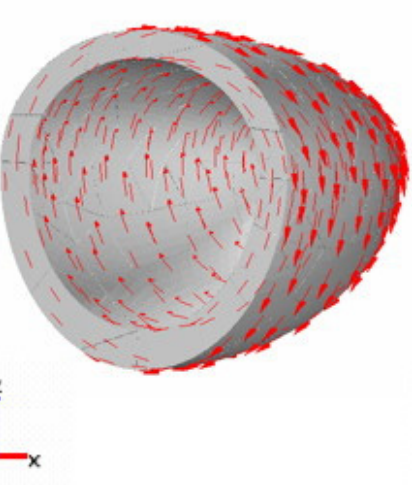

(b)

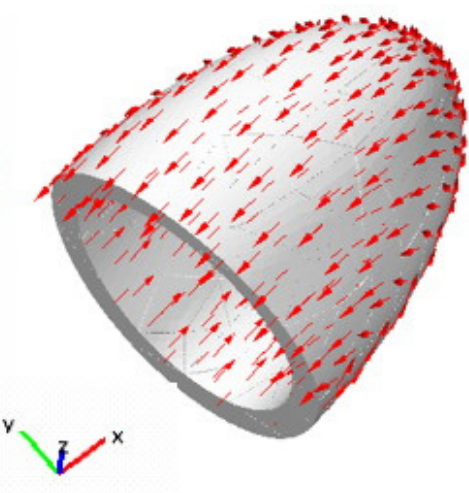

(c)

Figure 3. The associated vectors to the three parameters at the initial configuration $\Omega_{0}: n_{0 \xi}$ (a), $\nabla \theta_{\xi}$ (b) and $\nabla \varphi_{\xi}$ (c) (for sake of clarity only half of the embryo geometry has been represented)

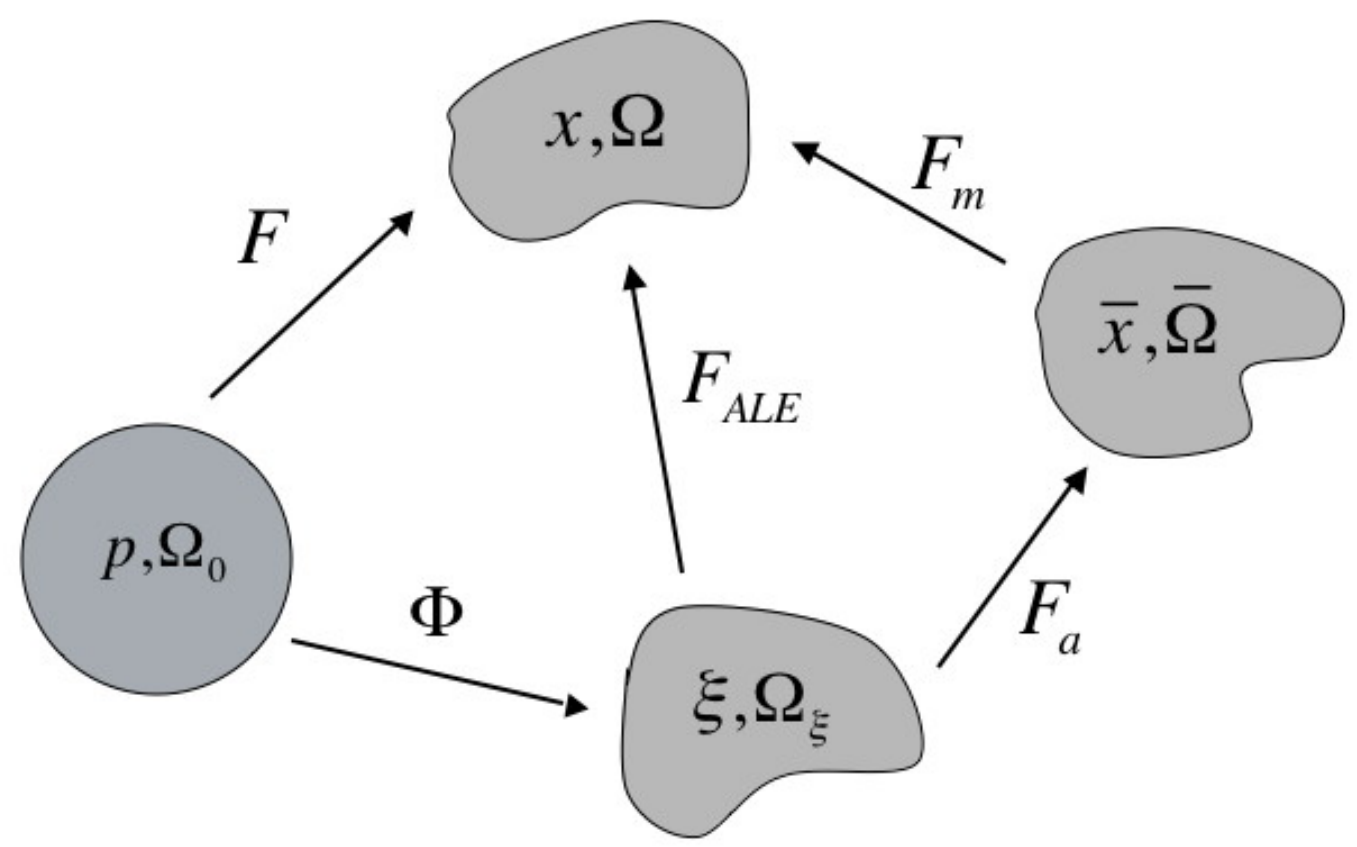

Figure 4. The diagram represents the successive configurations of the concurrent simulation. $\Omega_{0}$ the initial configuration, $\Omega_{\xi}$ the ALE configuration, $\bar{\Omega}$ the intermediate configuration and $\Omega$ the final configuration 


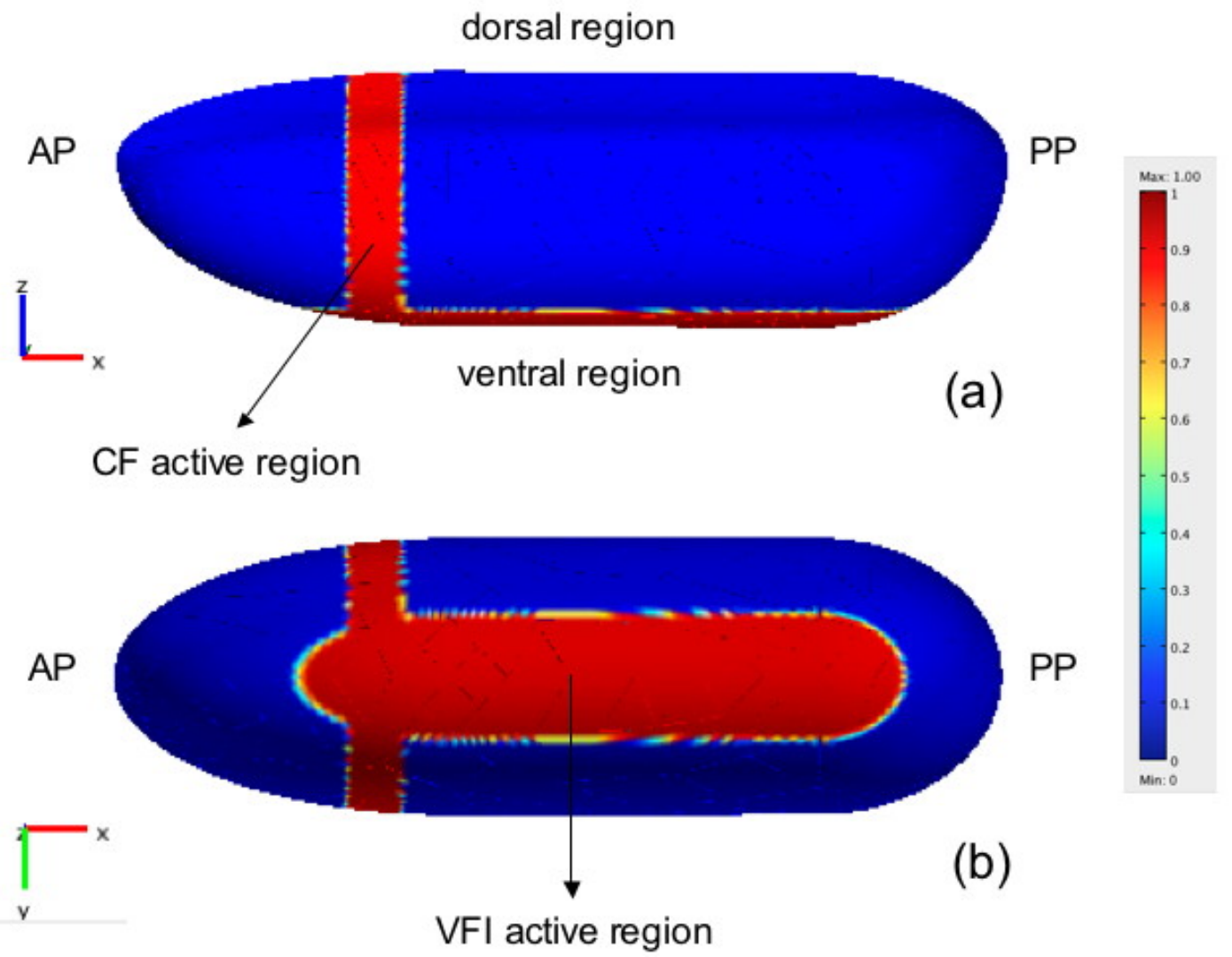

Figure 5. In red the active regions of the cephalic and the ventral furrows (the colors bar indicates the value of the characteristic fonction defining the active region (Allena et al, 2010a)). (a) Frontal view of the embryo. (b) Bottom view of the embryo

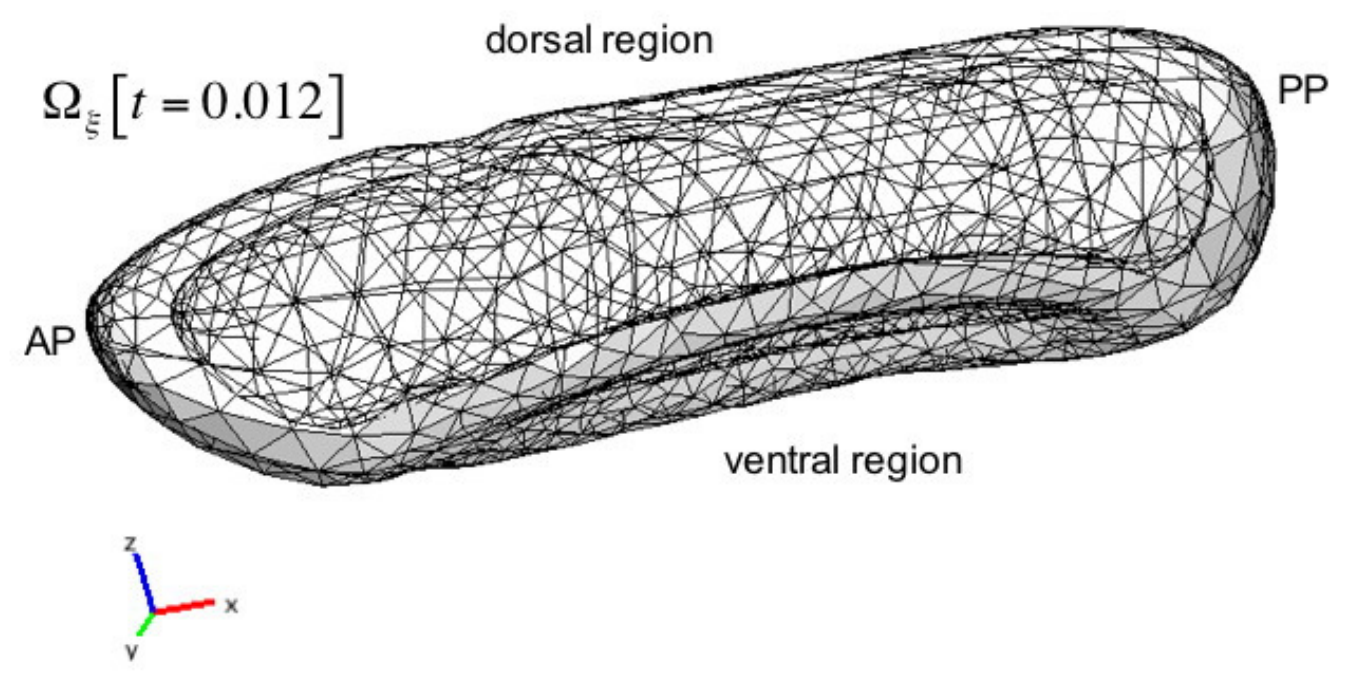

Figure 6. The embryo geometry at the end of the formation of the cephalic and the ventral furrows. This is the reference configuration with respect to which the elementary deformations responsible for the GBE are described 


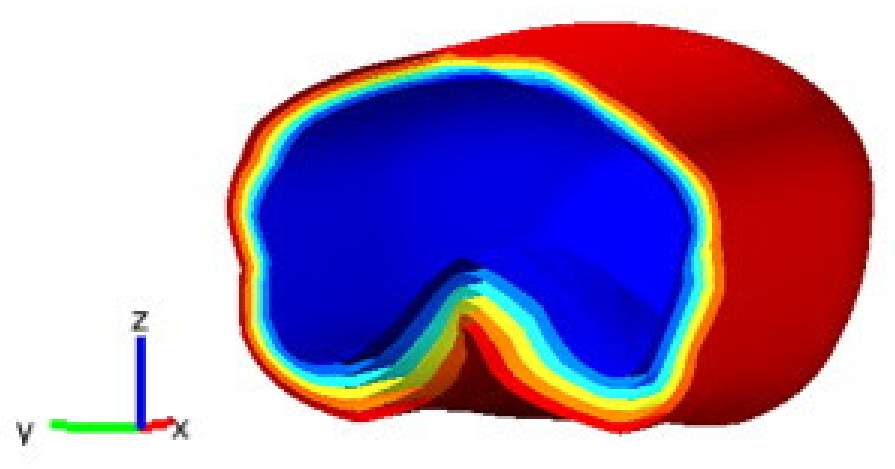

(a)
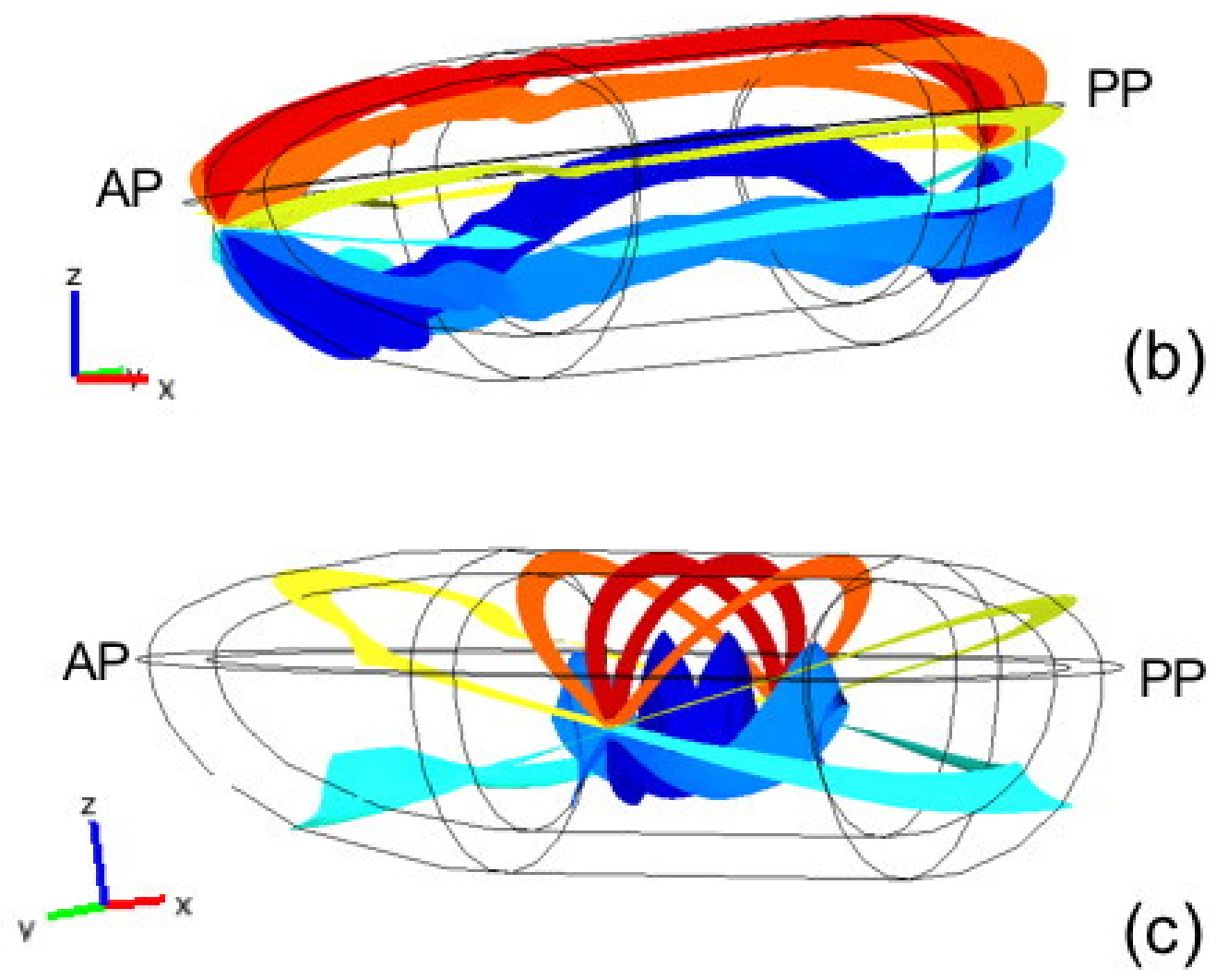

Figure 7. The isovalues of the three parameters obtained by the harmonic parametrization of the geometry at the particular intermediate configuration $\Omega_{\xi}[\mathrm{t}=0.012] .: \zeta_{\xi}(\mathrm{a}), \theta_{\xi}(\mathrm{b})$ and $\varphi_{\xi}(\mathrm{c})$ 


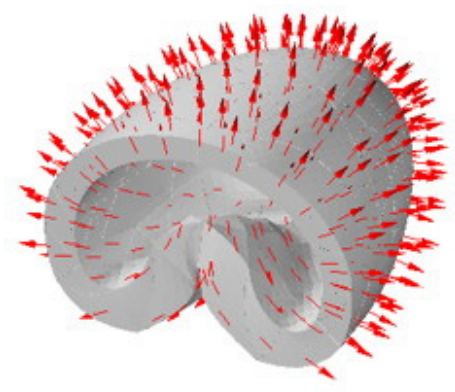

$y=1^{x}$

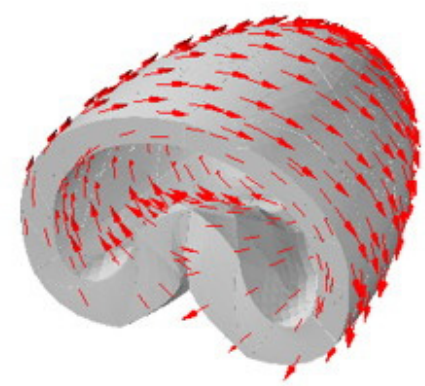

$y=\sum^{x}$

(a)

(b)

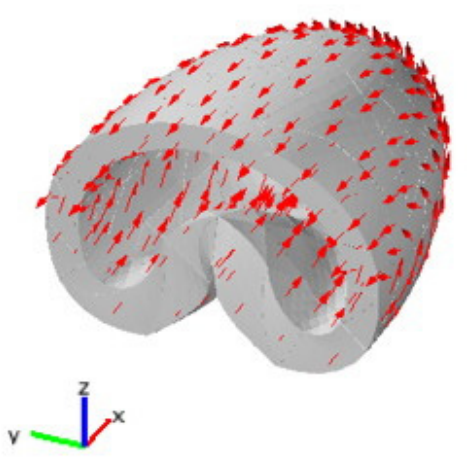

(c)

Figure 8 . The associated vectors to the three parameters at the particular intermediate configuration $\Omega_{\xi}[t=0.012]$ : $n_{0 \xi}(\mathrm{a}), \nabla \theta_{\xi}(\mathrm{b})$ and $\nabla \varphi_{\xi}$ (c)

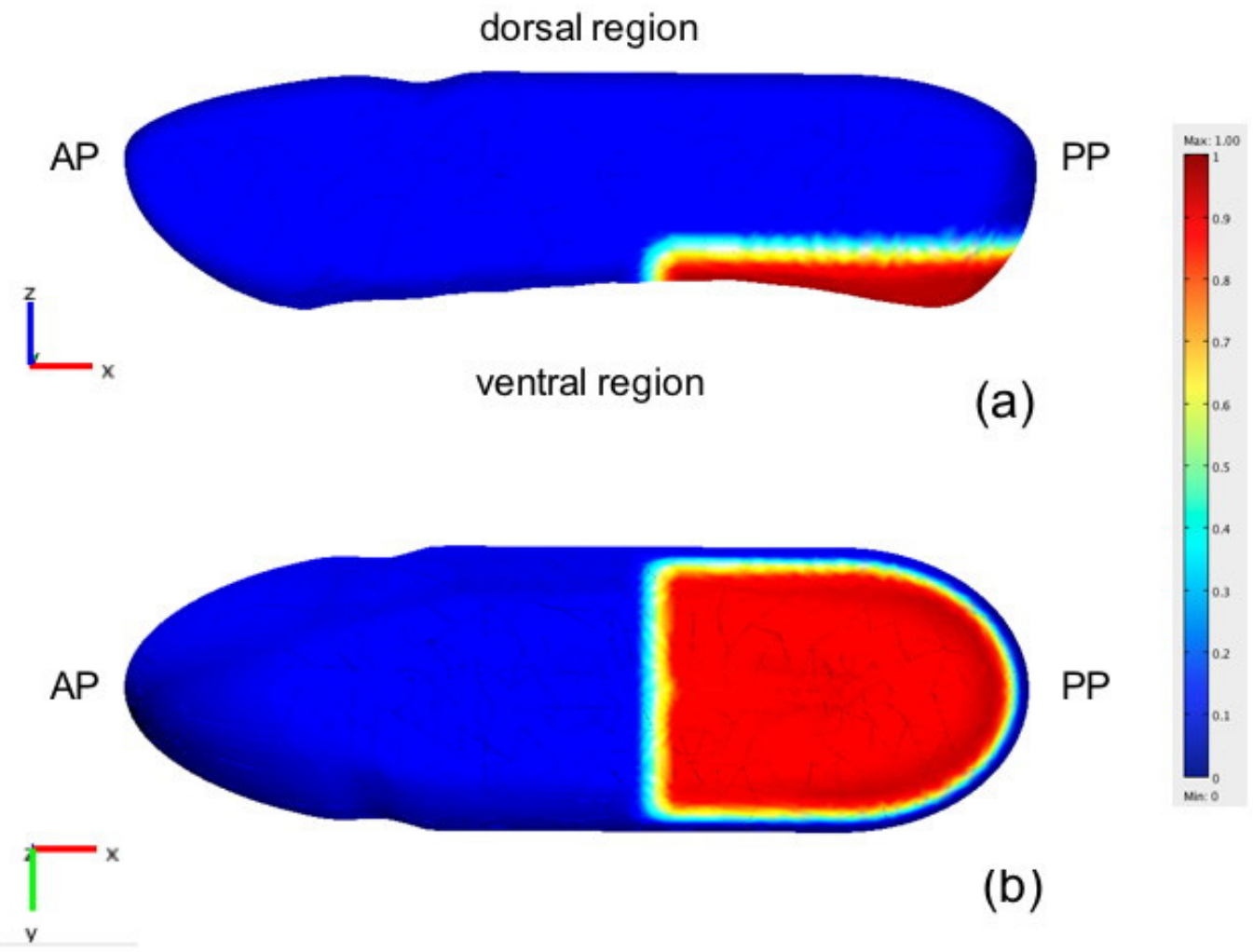

Figure 9. In red the active region for the GBE (the colors bar indicates the value of the characteristic fonction defining the active region (Allena et al, 2010a)). (a) Frontal view of the embryo. (b) Bottom view of the embryo 


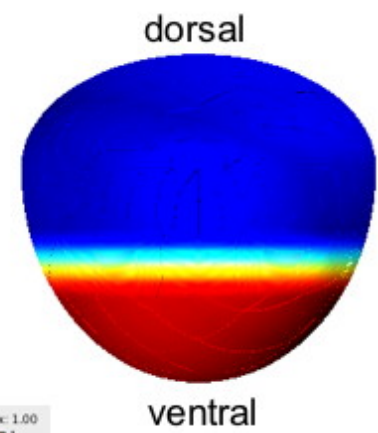

(a) $t=0$

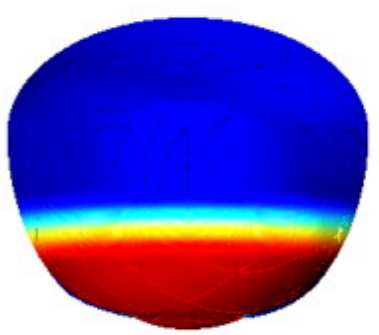

(b) $\mathrm{t}=0.006$

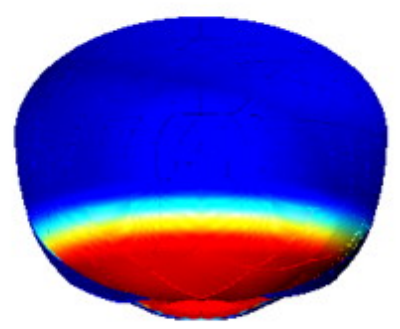

(c) $t=0.012$

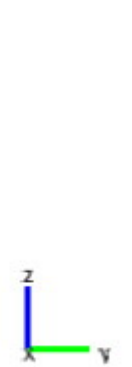

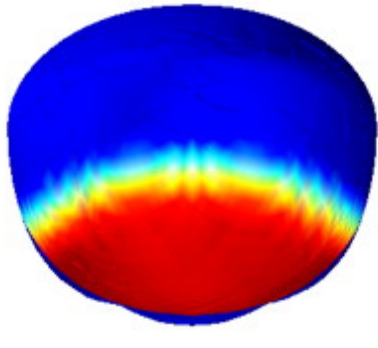

(d) $t=0.1$

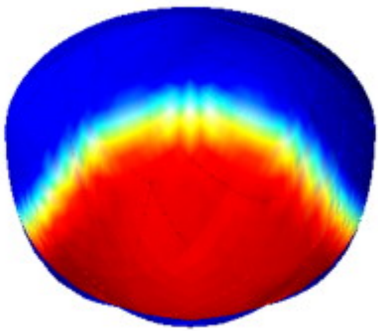

(e) $t=0.214$

Figure 10. Successive steps of the concurrent simulation from the rear point of view of the embryo. It is possible to observe the elongation of the GBE active region (red) towards the upper posterior pole (c :e) (the colors bar indicates the value of the characteristic fonction defining the active region (Allena et al, 2010a)) 


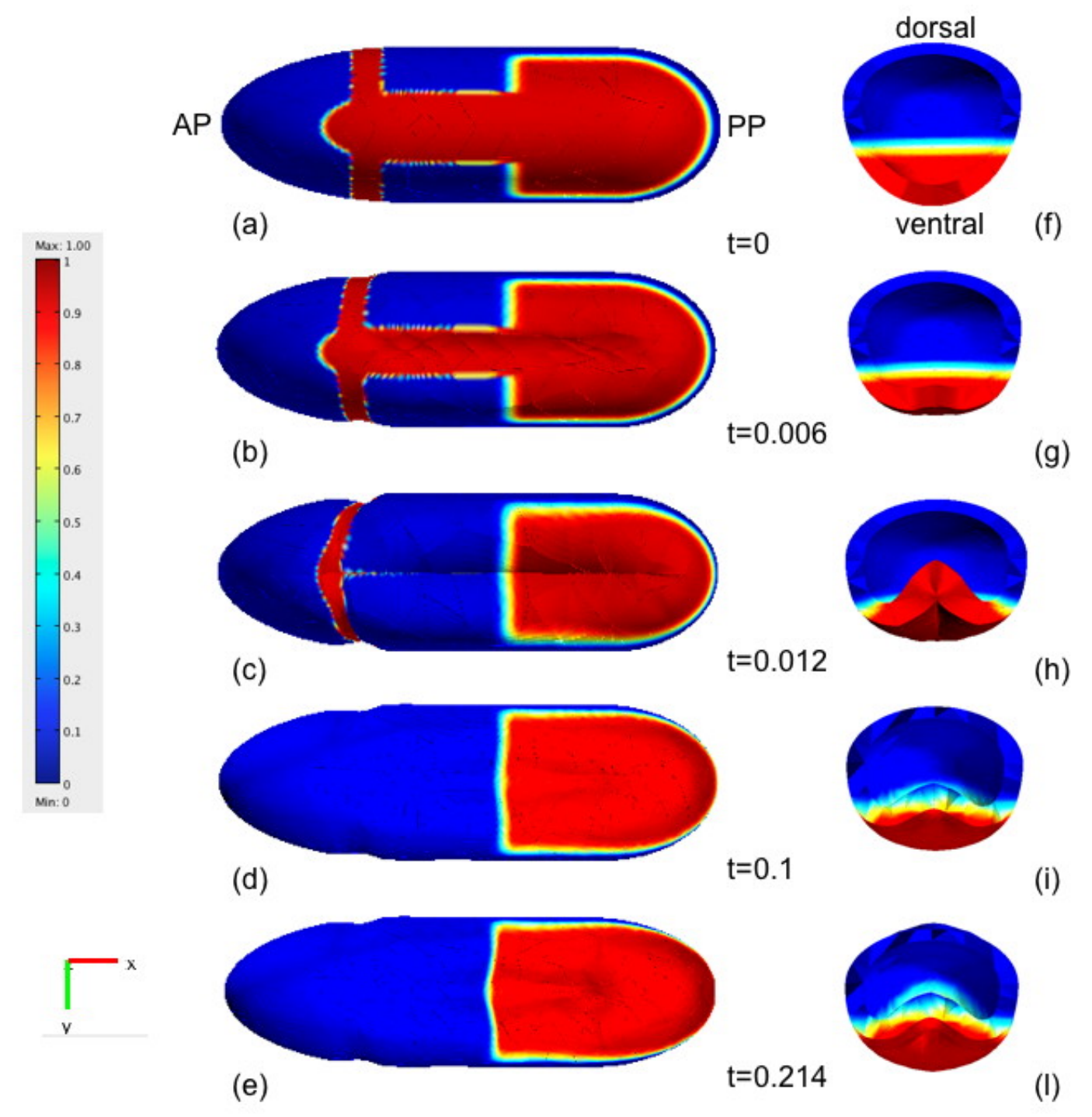

Figure 11. Successive steps of the concurrent simulation from the bottom point of view (a :e) and as cross sections (f :l) of the embryo. The CF and the VFI active regions (red) are represented until the two furrows have completely formed $(\mathrm{a}: \mathrm{c}, \mathrm{f}: \mathrm{h})$. The GBE active region (red) is represented all over the simulation $(\mathrm{a}: \mathrm{e}, \mathrm{f}: \mathrm{l})$ and the elongation of the ventral tissue towards the posterior pole is observed in (d) and (e) (the colors bar indicates the value of the characteristic fonction defining the active region (Allena et al, 2010a)) 


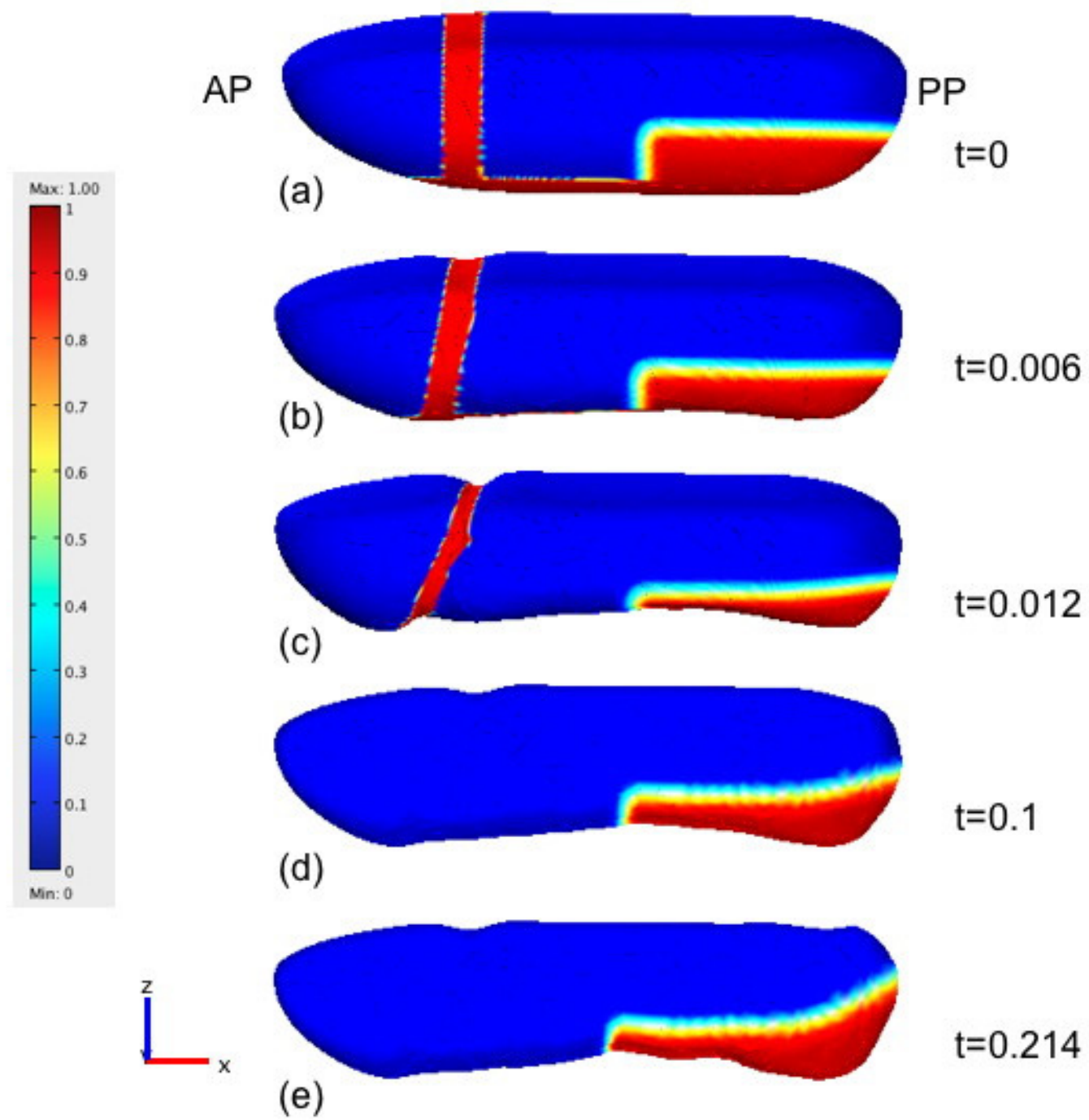

Figure 12. Successive steps of the concurrent simulation from the frontal point of view of the embryo. Again, the $\mathrm{CF}$ and the VFI active regions are represented in red until the complete formation of the two furrows. However, the GBE active region (red) is represented all over the simulation (the colors bar indicates the value of the characteristic fonction defining the active region (Allena et al, 2010a)) 


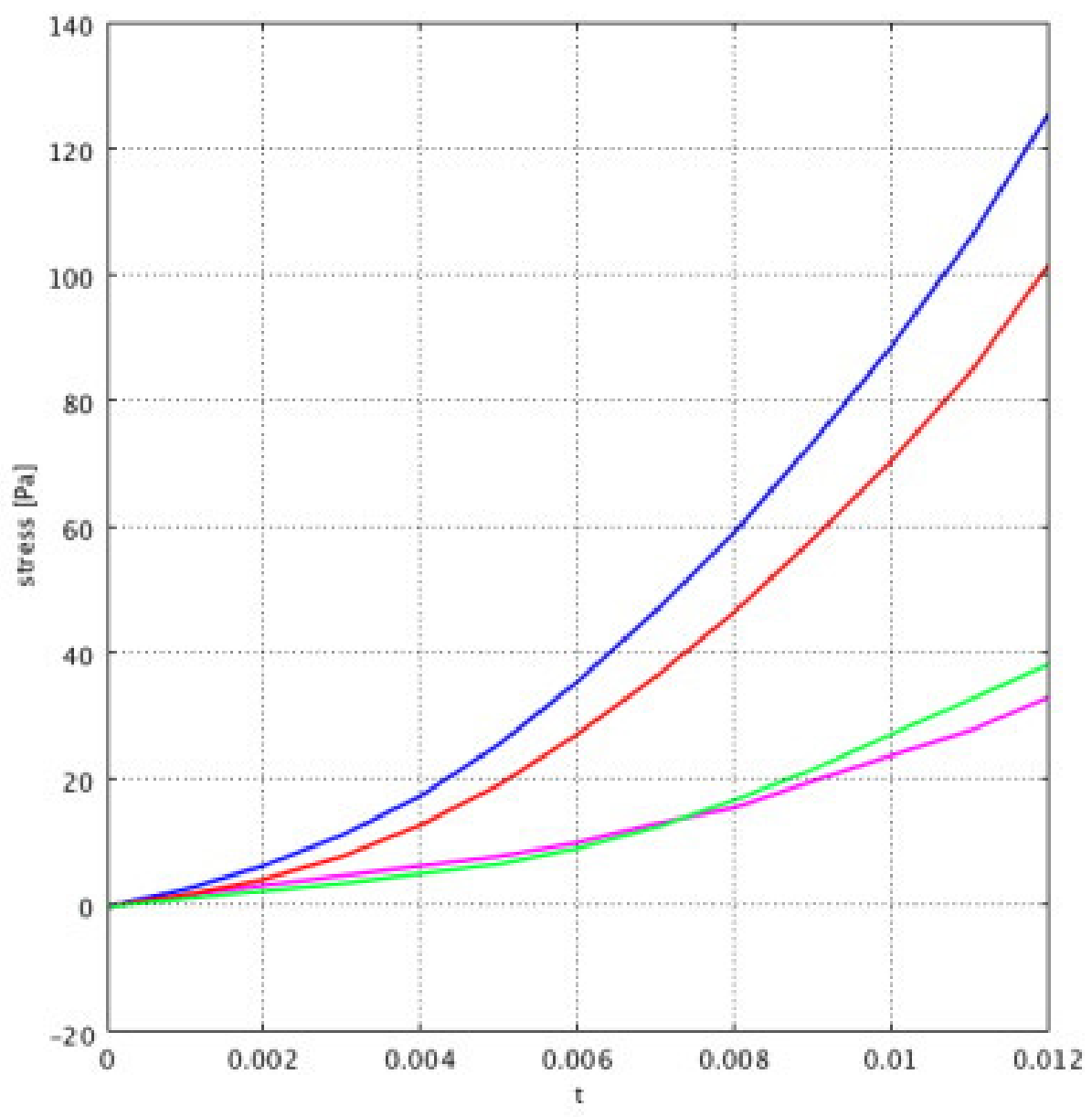

Figure 13. Variation of $\sigma_{\varphi \xi \varphi \xi}$ within the VFI active region over the first phase of the concurrent simulation $(0<t<0.012)$. We have evaluated $\sigma_{\varphi \xi \xi \xi}$ at both the anterior and the posterior frontiers of the VFI active region

(Fig. 5b). In particular, we have considered the four boundaries of the area: the anterior outside (blue), the posterior outside (red), the anterior inside (purple) and the posterior inside (green) boundaries 